\title{
The Polymer Incarcerated Method for the Preparation of Highly Active Heterogeneous Palladium Catalysts
}

\author{
Ryo Akiyama and Shū Kobayashi* \\ Graduate School of Pharmaceutical Sciences, The University of Tokyo, Hongo, \\ Bunkyo-ku, Tokyo 113-0033, Japan
}

\section{Supporting Information}

Preparation of tetraethyleneglycol mono-2-phenyl-2-propenyl ether (3): To sodium hydride (60\% in mineral oil, $1.82 \mathrm{~g}, 45.4 \mathrm{mmol})$ suspended in tetrahydrofurane $(70 \mathrm{~mL})$, tetraethyleneglycol $(8.81 \mathrm{~mL}, 45.4 \mathrm{mmol})$ was added at 0 ${ }^{\circ} \mathrm{C}$. After the reaction mixture was stirred for $1 \mathrm{~h}$ at room temperature, 3-chloro-2phenylpropene ( $3.46 \mathrm{~g}, 22.7 \mathrm{mmol}$ ) was added and the mixture was further stirred for $12 \mathrm{~h}$. After the mixture was cooled to $0{ }^{\circ} \mathrm{C}$ and diluted with diethyl ether, saturated aqueous ammonium chloride was added to quench the reaction and the aqueous layer was extracted with diethyl ether. The combined organic layers were dried over sodium sulfate and the solvent was removed under reduced pressure. The residue was purified by silica gel column chromatography to afford tetraethyleneglycol mono-2phenyl-2-propenyl ether $(3,4.52 \mathrm{~g}, 64 \%)$ and tetraethyleneglycol di-2-phenyl-2propenyl ether_621 mg, 13\%).

Tetraethyleneglycol mono-2-phenyl-2-propenyl ether (3): ${ }^{1} \mathrm{H}$ NMR $\delta=2.72$ (s, $1 \mathrm{H}), 3.58-3.74(\mathrm{~m}, 16 \mathrm{H}), 4.42(\mathrm{~s}, 2 \mathrm{H}), 5.34(\mathrm{~d}, 1 \mathrm{H}, J=1.2 \mathrm{~Hz}), 5.53(\mathrm{~d}, 1 \mathrm{H}, J=0.5$ $\mathrm{Hz}), 7.25-7.36(\mathrm{~m}, 3 \mathrm{H}), 7.44-7.52(\mathrm{~m}, 2 \mathrm{H}) ;{ }^{13} \mathrm{C} \mathrm{NMR}=61.7,69.2,70.3,70.53$, 70.58, 72.4, 73.1, 114.4, 126.1, 127.7, 128.3, 138.7, 144.0.

Tetraethyleneglycol di-2-phenyl-2-propenyl ether: ${ }^{1} \mathrm{H}$ NMR $\delta=3.55-3.75(\mathrm{~m}$, $16 \mathrm{H}), 4.41(\mathrm{~s}, 4 \mathrm{H}), 5.33(\mathrm{~d}, 2 \mathrm{H}, J=1.0 \mathrm{~Hz}), 5.51(\mathrm{~s}, 2 \mathrm{H}), 7.23-7.36(\mathrm{~m}, 6 \mathrm{H}), 7.43-$ $7.50(\mathrm{~m}, 4 \mathrm{H}) ;{ }^{13} \mathrm{C} \mathrm{NMR}=69.2,70.47,70.51,73.0,114.3,126.0,127.6,128.2,138.7$, 144.0.

Preparation of copolymer 1c: Styrene (12.3 g, $118 \mathrm{mmol})$, 4-vinylbenzyl glycidy ether (2.82 g, $14.8 \mathrm{mmol})$, tetraethyleneglycol mono-2-phenyl-2-propenyl ether (3, $4.52 \mathrm{~g}, 14.6 \mathrm{mmol}$ ), and 2,2'-azobis(isobutyronitrile) (172 $\mathrm{mg}, 1.05 \mathrm{mmol}$ ) were mixed in chloroform $(52.5 \mathrm{~mL})$. The mixture was stirred for $48 \mathrm{~h}$ under reflux conditions and then cooled to room temperature. The resulting polymer solution was pored slowly into cooled methanol. The precipitated polymer was filtered and 
washed with methanol several times and dried for $24 \mathrm{~h}$ under reduced pressure to afford the desired copolymer (1c, $10.4 \mathrm{~g}, 53 \%$ yield). The molar ratio of the monomer was determined by ${ }^{1} \mathrm{H}$ NMR analysis (styrene : 4-vinylbenzyl glycidy ether : tetraethyleneglycol mono-2-phenyl-2-propenyl ether $=90: 4: 6) . M_{w}: 69,985, M_{n}$ : 12,098, $\mathrm{M}_{\mathrm{W}} / \mathrm{M}_{\mathrm{n}}=5.785(\mathrm{GPC})$.

Preparation of polymer incarcerated palladium (PI Pd, 4c, Figure 1): Copolymer (1c, $1.00 \mathrm{~g})$ was dissolved in tetrahydrofuran $(20 \mathrm{~mL})$ at room temperature, and to this solution was added tetrakis(triphenylphosphine)palladium(0) $\left(\mathrm{Pd}\left(\mathrm{PPh}_{3}\right)_{4}, 0.10 \mathrm{~g}\right)$ as a core $\left(\mathrm{Pd}\left(\mathrm{PPh}_{3}\right)_{4}\right.$ was dissolved $)$. The mixture was stirred for $24 \mathrm{~h}$ at this temperature. Hexane $(30 \mathrm{~mL})$ was slowly added to the mixture at $0{ }^{\circ} \mathrm{C}$. Coaservates were found to envelop the core dispersed in the medium. The mixture was left to stand at room temperature for $12 \mathrm{~h}$, and the catalyst capsules were then washed with hexane several times and dried at room temperature for $24 \mathrm{~h}$. Next, the catalyst capsules were stirred at $120{ }^{\circ} \mathrm{C}$ for $2 \mathrm{~h}$ to prepare cross-linked microencapsulated palladium. The crosslinked catalyst was then washed with tetrahydrofuran several times and dried at room temperature for $24 \mathrm{~h}$ to give polymer incarcerated palladium ${ }^{1}$ (PI Pd, 4c, $792 \mathrm{mg}$, $0.108 \mathrm{mmol} / \mathrm{g}, 97 \%$ of palladium metal was loaded). The washings were concentrated to determine loading of palladium metal by fluorescence X-ray analysis. Four equivalents of triphenylphosphine $\left(\mathrm{PPh}_{3}\right)$ were recovered from the washings.

Hydrogenation of benzalacetone using PI Pd 4c (Table 1): A typical experimental procedure is described for the hydrogenation of benzalacetone. PI Pd (4c, 0.025 mmol, $5 \mathrm{~mol} \mathrm{\%}$ ) and benzalacetone $(73.0 \mathrm{mg}, 0.50 \mathrm{mmol})$ were combined in tetrahydrofuran $(5 \mathrm{~mL})$. The mixture was stirred for $1 \mathrm{~h}$ at room temperature and atmosphiore of hydrogen (1 atm). After hexane was added to quench the reaction, the catalyst was filtered and washed with tetrahydrofuran, and then dried. The solvents of the filtrate were removed under reduced pressure, and the crude product was purified by preparative TLC to afford 4-phenyl-2-butanone $(\mathbf{5}, 60.0 \mathrm{mg}, 81 \%)$ and 4-phenyl-2butanol $(\mathbf{6}, 3.0 \mathrm{mg}, 4 \%)$. The recovered $\mathbf{4 c}$ was reused several times without loss of activity.

4-Phenyl-2-butanone (5): ${ }^{1} \mathrm{H}$ NMR $\left(\mathrm{CDCl}_{3}\right) \delta=2.12(\mathrm{~s}, 3 \mathrm{H}), 2.74(\mathrm{t}, 2 \mathrm{H}, J=7.7$ $\mathrm{Hz}), 2.88(\mathrm{t}, 2 \mathrm{H}, J=7.7 \mathrm{~Hz}), 7.10-7.30(\mathrm{~m}, 5 \mathrm{H}) ;{ }^{13} \mathrm{C} \mathrm{NMR}\left(\mathrm{CDCl}_{3}\right) \delta=29.6,29.9$, 45.0, 126.0, 128.2, 128.4, 140.9, 207.8.

4-Phenyl-2-butanol (6): ${ }^{1} \mathrm{H} \mathrm{NMR}\left(\mathrm{CDCl}_{3}\right) \delta=1.23$ (d, 3H, $\left.J=6.3 \mathrm{~Hz}\right), 1.47$ (brs, $1 \mathrm{H}), 1.70-1.84(\mathrm{~m}, 2 \mathrm{H}), 2.55-2.85(\mathrm{~m}, 2 \mathrm{H}), 3.75-3.90(\mathrm{~m}, 2 \mathrm{H}) .7 .15-7.33(\mathrm{~m}, 5 \mathrm{H}) ;{ }^{13} \mathrm{C}$ $\operatorname{NMR}\left(\mathrm{CDCl}_{3}\right) \delta=23.7,32.1,40.9,125.8,128.4,124.0$.

1,2-Diphenylethane: ${ }^{1} \mathrm{H}$ NMR $\left(\mathrm{CDCl}_{3}\right) \delta=2.90(\mathrm{~s}, 4 \mathrm{H}), 7.10-7.29(\mathrm{~m}, 10 \mathrm{H}) ;{ }^{13} \mathrm{C}$ 
$\operatorname{NMR}\left(\mathrm{CDCl}_{3}\right) \delta=37.9,125.9,128.3,128.4,141.7$.

1,4-Diphenylbutane: ${ }^{1} \mathrm{H} \mathrm{NMR}\left(\mathrm{CDCl}_{3}\right) \delta=1.65(\mathrm{~m}, 4 \mathrm{H}), 2.61(\mathrm{~m}, 4 \mathrm{H}), 7.10-7.30$ (m, $10 \mathrm{H})$.

$N$-Benzoylphenylalanine methyl ester: ${ }^{1} \mathrm{H}$ NMR $\left(\mathrm{CDCl}_{3}\right) \delta=3.20(\mathrm{dd}, 1 \mathrm{H}, J=5.7$, $13.7 \mathrm{~Hz}), 3.28(\mathrm{dd}, 1 \mathrm{H}, J=5.7,13.7 \mathrm{~Hz}), 3.74(\mathrm{~s}, 3 \mathrm{H}), 5.08(\mathrm{dt}, 1 \mathrm{H}, J=5.7,7.5 \mathrm{~Hz})$, 6.71 (brd, $1 \mathrm{H}, J=7.5 \mathrm{~Hz}), 7.10-7.52(\mathrm{~m}, 8 \mathrm{H}), 7.68-7.76(\mathrm{~m}, 2 \mathrm{H}) ;{ }^{13} \mathrm{C} \mathrm{NMR}\left(\mathrm{CDCl}_{3}\right)$ $\delta=37.7,52.3,53.5,126.9,127.0,128.47,128.49,129.2,131.6,133.8,135.8,166.8$, 172.0 .

1,2-Dipropoxybenzene: ${ }^{1} \mathrm{H}$ NMR $\left(\mathrm{CDCl}_{3}\right) \delta=1.04(\mathrm{t}, 6 \mathrm{H}, J=4.4 \mathrm{~Hz}), 1.82(\mathrm{~m}$, $4 \mathrm{H}), 3.96(\mathrm{t}, 4 \mathrm{H}, J=6.6 \mathrm{~Hz}), 6.89(\mathrm{~s}, 4 \mathrm{H}) ;{ }^{13} \mathrm{C} \mathrm{NMR}\left(\mathrm{CDCl}_{3}\right) \delta=10.4,22.6,70.7$, 114.1, 121.0, 149.2.

\section{Details of Allylation reaction of allyl methyl carbonate (7) with dimethyl phenylmalonate $(8)$ :}

PI Pd (4a-c) were applied to allylic substitution reactions, one of representative carbon-carbon bond-forming reactions. We chose the reaction of allyl methyl carbonate (7) with dimethyl phenylmalonate $(\mathbf{8})$ as a model, and several reaction conditions were examined (Table S-1). It was found that the allylation reaction proceeded in the presence of $\mathrm{PI} \mathrm{Pd}(5 \mathrm{~mol} \%)$ and $\mathrm{PPh}_{3}(5 \mathrm{~mol} \%)$ in THF under reflux conditions. While $\mathbf{4 a}$ gave lower yields, the activity of $\mathbf{4 b}$ and $\mathbf{4 c}$ was high, and the desired allylation adducts were obtained in high yields. The catalysts were recovered by simple filtration and reused several times. In the case of $\mathbf{4 b}$, however, leaching of palladium from $\mathbf{4 b}$ occurred in the fourth and the fifth use. On the other hand, no leaching of palladium was observed in the reactions using $\mathbf{4 c}$ even after the fifth use, and the reactions proceeded smoothly to afford the desired adducts in high yields in all cases.

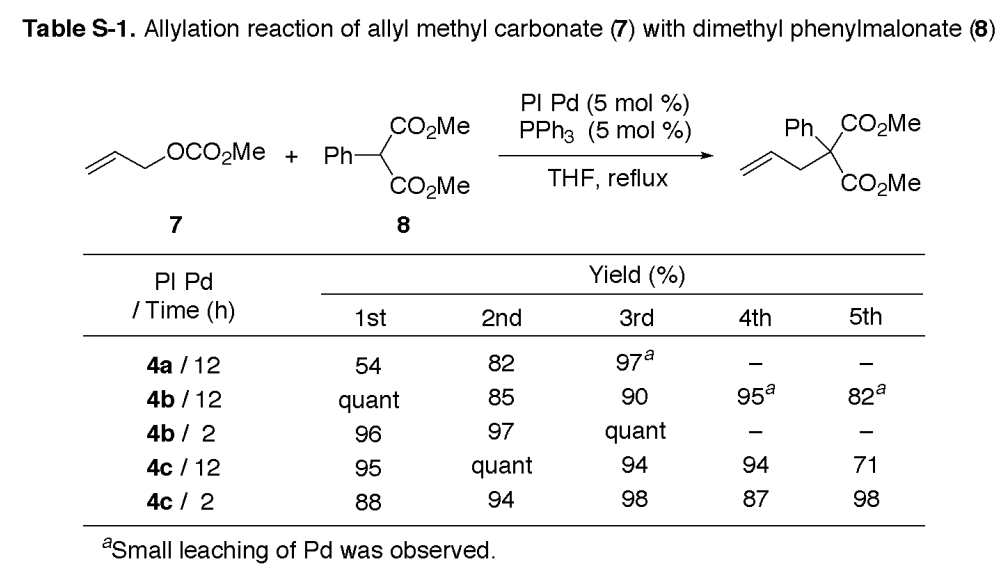


Allylation reaction using PI Pd(0) 4c (Table 2): A typical experimental procedure is described for the allylation reaction of allyl methyl carbonate (7) with dimethyl phenylmalonate (8): 7 (63.9 mg, $0.55 \mathrm{mmol}), 8$ (104.1 mg, $0.50 \mathrm{mmol}), \mathrm{PPh}_{3}$ (26.3 mg, $0.1 \mathrm{mmol}, 20 \mathrm{~mol} \%$ ), and PI Pd (4c, $0.025 \mathrm{mmol}, 5 \mathrm{~mol} \%)$ were combined in tetrahydrofuran $(5 \mathrm{~mL})$. The mixture was stirred for $12 \mathrm{~h}$ at room temperature. After ethanol was added to quench the reaction, the catalyst was filtered and washed with ethanol and acetonitrile, and then dried. The solvents of the filtrate were removed under reduced pressure, and the crude product was purified by preparative TLC to afford dimethyl allylphenylmalonate $(109.3 \mathrm{mg}, 88 \%)$. The recovered $\mathbf{4 c}$ was reused several times without loss of activity.

Dimethyl allylphenylmolonate: ${ }^{1} \mathrm{H} \mathrm{NMR}\left(\mathrm{CDCl}_{3}\right) \delta=3.07(\mathrm{~d}, 2 \mathrm{H}, J=7.1 \mathrm{~Hz}), 3.72$ (s, 6H), 5.02-5.11 (m, 2H), 5.77 (ddt, $1 \mathrm{H}, J=7.1,10.1,17.1 \mathrm{~Hz}), 7.24-7.42(\mathrm{~m}, 5 \mathrm{H})$; ${ }^{13} \mathrm{C} \mathrm{NMR}\left(\mathrm{CDCl}_{3}\right) \delta=40.2,53.5,62.9,118.7,127.6,127.8,128.1,132.9,136.6$, 170.6; IR (neat) $1736 \mathrm{~cm}^{-1}$; Elemental anal. Calcd for $\mathrm{C}_{14} \mathrm{H}_{16} \mathrm{O}_{4}$ : C 67.73, H 6.50; found: C 67.57, H 6.52 .

Dimethyl methallylphenylmolonate: ${ }^{1} \mathrm{H} \mathrm{NMR}\left(\mathrm{CDCl}_{3}\right) \delta=1.57(\mathrm{~s}, 3 \mathrm{H}), 3.13(\mathrm{~s}$, $2 \mathrm{H}), 3.71(\mathrm{~s}, 6 \mathrm{H}), 4.55(\mathrm{~m}, 1 \mathrm{H}), 4.78(\mathrm{~m}, 1 \mathrm{H}), 7.23-7.36(\mathrm{~m}, 3 \mathrm{H}), 7.46-7.53(\mathrm{~m}, 2 \mathrm{H})$; ${ }^{13} \mathrm{C} \mathrm{NMR}\left(\mathrm{CDCl}_{3}\right) \delta=23.6,43.3,52.5,62.3,115.1,127.5,127.9,128.0,136.7$, 140.9, 170.7; IR (neat) $1737 \mathrm{~cm}^{-1}$; Elemental anal. Calcd for $\mathrm{C}_{15} \mathrm{H}_{18} \mathrm{O}_{4}: \mathrm{C} 68.68, \mathrm{H}$ 6.92; found: C 68.42, H 6.82.

Ethyl 2-acethyl-5-phenyl-4-pentenoate ${ }^{2}$ : diastereomer mixture (64:36); ${ }^{1} \mathrm{H}$ NMR $\left(\mathrm{CDCl}_{3}\right) \delta=1.24(\mathrm{t}, 3 \mathrm{H}, J=7.1 \mathrm{~Hz}), 2.19(\mathrm{~s}, 1.9 \mathrm{H}), 2.24(1.1 \mathrm{H}), 2.68-2.91(\mathrm{~m}, 3 \mathrm{H})$, 4.15-4.25 (m, 2H), 5.95-6.20 (m, 1H), $6.45(\mathrm{~d}, 1 \mathrm{H}, J=15.7 \mathrm{~Hz}), 7.15-5.40(\mathrm{~m}, 5 \mathrm{H})$; ${ }^{13} \mathrm{C} \mathrm{NMR}\left(\mathrm{CDCl}_{3}\right) \delta=14.0,14.1,27.1,29.1,31.4,35.7,59.5,61.37,61.41,63.8$, $123.7,125.7,126.07,126.14,1127.3,127.4,128.0,128.4,128.5,132.6,134.0,136.9$, 169.1, 171.4, 202.3, 204.1.

2-Allyl-2-methoxycarbonyl-1-indanone ${ }^{3}:{ }^{1} \mathrm{H}$ NMR $\left(\mathrm{CDCl}_{3}\right) \delta=2.62(\mathrm{ddt}, 1 \mathrm{H}, J=$ 1.0, 7.3, 13.9 Hz), 2.89 (dd, $1 \mathrm{H}, J=7.3,13.9 \mathrm{~Hz}), 3.16(\mathrm{~d}, 1 \mathrm{H}, J=17.4 \mathrm{~Hz}), 3.66$ (d, $1 \mathrm{H}, J=17.4 \mathrm{~Hz}$ ), $3.68(\mathrm{~s}, 3 \mathrm{H}), 5.00-5.20(\mathrm{~m}, 2 \mathrm{H}), 5.64(\mathrm{ddt}, 1 \mathrm{H}, J=7.3,10.1,16.9$ $\mathrm{Hz}), 7.35-7.80(\mathrm{~m}, 4 \mathrm{H}) ;{ }^{13} \mathrm{C} \mathrm{NMR}\left(\mathrm{CDCl}_{3}\right) \delta=35.8,36.0,38.9,52,6,59.7,119.2$, $124.5,126.3,127.6,132.5,134.9,135.3,152.9,171.1,201.9$.

1-(Allyloxy)naphthalene ${ }^{4}:{ }^{1} \mathrm{H}$ NMR $\left(\mathrm{CDCl}_{3}\right) \delta=4.63(\mathrm{~d}, 2 \mathrm{H}, J=5.1 \mathrm{~Hz}), 5.29$ (dd, $1 \mathrm{H}, J=1.6,10.4 \mathrm{~Hz}), 5.48(\mathrm{dd}, 1 \mathrm{H}, J=1.6,17.2 \mathrm{~Hz}), 6.12(\mathrm{ddt}, 1 \mathrm{H}, J=5.1,10.4$, $17.2 \mathrm{~Hz}), 6.73$ (d, $1 \mathrm{H}, J=7.5 \mathrm{~Hz}), 7.25-7.50(\mathrm{~m}, 4 \mathrm{H}), 7.70-7.80(\mathrm{~m}, 1 \mathrm{H}), 8.25-8.35$ $(\mathrm{m}, 1 \mathrm{H}) ;{ }^{13} \mathrm{C} \mathrm{NMR}\left(\mathrm{CDCl}_{3}\right) \delta=68.8,105.0,117.2,120.3,122.1,125.1,125.72$, $125.74,126.3,127.4,133.3,134.5,154.2$.

2-(Allyloxy)naphthalene ${ }^{5}:{ }^{1} \mathrm{H}$ NMR $\left(\mathrm{CDCl}_{3}\right) \delta=4.59(\mathrm{~d}, 2 \mathrm{H}, J=5.4 \mathrm{~Hz}), 5.29(\mathrm{~d}$, $1 \mathrm{H}, J=10.5 \mathrm{~Hz}), 5.43$ (d, 1H, $J=17.4 \mathrm{~Hz}), 6.08$ (ddt, $1 \mathrm{H}, J=5.4,10.5,17.4 \mathrm{~Hz}$ ), 
7.10-7.20 (m, 2H), 7.30-7.45 (m, 2H), 7.65-7.75 (m, 3H); ${ }^{13} \mathrm{C} \mathrm{NMR}\left(\mathrm{CDCl}_{3}\right) \delta=$ 68.7, 106.9, 117.7, 118.9, 123.6, 126.3, 126.7, 127.6, 129.0, 129.4, 133.1, 134.5, 156.5 .

3-(4-Nitrophenyloxy)-1-propene': ${ }^{1} \mathrm{H} \mathrm{NMR}\left(\mathrm{CDCl}_{3}\right) \delta=4.64(\mathrm{~d}, 2 \mathrm{H}, J=5.3 \mathrm{~Hz})$, $5.35(\mathrm{~d}, 1 \mathrm{H}, J=10.4 \mathrm{~Hz}), 5.44(\mathrm{~d}, 1 \mathrm{H}, J=17.2 \mathrm{~Hz}), 6.05$ (ddt, $1 \mathrm{H}, J=5.3,10.4,17.2$ $\mathrm{Hz}), 6.97(2 \mathrm{H}, J=9.0 \mathrm{~Hz}), 8.18(2 \mathrm{H}, J=9.0 \mathrm{~Hz}) ;{ }^{13} \mathrm{C} \mathrm{NMR}\left(\mathrm{CDCl}_{3}\right) \delta=69.3,114.6$, $118.5,125.7,131.8,141.4,163.5$.

\section{References and Note}

1) As shown in the text, this term is inspired by the elegant work of Cram on carcerands, hemicarcerands, and their complexes. We are grateful to Professor Craig A. Merlic (UCLA) for his suggestion of this term. For the related references of carcerands, hemicarcerands, and their complexes, (a) Cram, D. J. Nature 1992, 356, 29. (b) Cram, D. J. Science 1983, 219, 1177. (c) Cram, D. J.; Karbach, S.; Kim, Y. H.; Baczynskyj, L.; Kalleymeyn, G. W. J. Am. Chem. Soc. 1985, 107, 2575. (d) Warmuth, R.; Yoon, J. Acc. Chem. Res. 2001, 34, 95. In addition, it was shown that some unstable species such as cyclobutadiene, obenzyne, and 1,2,4,6-cycloheptatetraene were stablized in carcerands and their complexes. (e) Cram, D. J.; Tanner, M. E.; Thomas, R. Angew. Chem. Int. Ed. 1991, 30, 1024. (f) Warmuth, R. Angew. Chem. Int. Ed. 1997, 36, 1347. (g) Warmuth, R.; Marvel, M. A. Angew. Chem. Int. Ed. 2000, 39, 1117.

2) S.-W., Zhang, T. Mitsudo, T. Kondo, Y. Watanabe, J. Organomet. Chem. 450, 197 (1993).

3) K. Ueemura, H. Matsuyama, N. Watanabe, M. Kobayashi, N. Kamigata, J. Org. Chem. 54, 2374 (1989).

4) C. Goux, M. Massacret, P. Lhoste, D. Sinou, Organometallics 14, 4585 (1995).

5) H. B. Mereyala, S. R. Lingannagaru, Tetrahedron 53, 17501 (1997).

6) W. N. White, D. Gwyn, R. Schlitt, C. Girard, W. Fife, J. Am. Chem. Soc. 80, 3271 (1958). 